\title{
Parameter Estimation of Bilinear Digital Systems Using Differential Evolution Algorithm
}

\author{
Wei-Der Chang $^{*} \quad$ Shun-Peng Shih Yi-Ting Hsiao Yu-Ting Xia Tien-Yu Liang Hao-Tse Huang \\ Department of Computer and Communication, Shu-Te University \\ Kaohsiung 824, Taiwan
}

*Corresponding Author: wdchang@stu.edu.tw

\begin{abstract}
In this paper, we propose a novel parameter estimation scheme for bilinear digital systems based on using the differential evolution (DE) algorithm. The DE algorithm is one of evolutionary computations and is fully with real-valued operations during the optimization. It has been proven to be an excellent searching algorithm in solving a variety of practical engineering optimization problems. With the use of the DE algorithm, the parameter estimation problem especially for the bilinear digital system is considered. By minimizing the error signal between the actual output and model output, the system coefficients can accurately be estimated. Finally, a large number of independent runs with different sets of initial conditions are examined to confirm the algorithm's robustness and feasibility.
\end{abstract}

Keywords: parameter estimation, bilinear digital system, differential evolution (DE).

\section{Introduction}

In recent years, many related researches and applications about the bilinear digital system have been developed and investigated such as nonlinear system modeling $^{(1)}$, parameter estimation ${ }^{(2,3)}$, system identification $^{(4-6)}$, and multichannel active control ${ }^{(7)}$. It is a nonlinear system and is also an extended version of a digital recursive filter, infinite impulse response (IIR) filter, which consists of three different kinds of series: input signals, output signals, and products of input and output signals. Thus, its modeling capacity is absolutely superior to the original IIR digital system, and conversely the complexity to design the bilinear digital system is more difficult than the IIR due to the nonlinearity.

To estimate the parameters of bilinear digital system, this paper is to utilize the differential evolution (DE) algorithm which belongs to one of evolutionary computations. This algorithm is a powerful and efficient searching means to solving the optimized problems. It is also a population-based algorithm with multiple direction searching. Recently, a variety of engineering optimization problems have been solved and explored by using the DE algorithm ${ }^{(1,8-11)}$. In [8], the authors proposed an optimization scheme which the DE is used to choose shape parameter and node distribution when applying the radial basis function meshless numerical method. In [10], a specialized DE technique to solve the transmission expansion planning (TEP) problem was developed and some comparisons were performed with other swarm methods. Moreover, an optimization problem of heliostat field layout in solar central receiver systems on annual basis has been solved using the DE algorithm ${ }^{(11)}$

This paper will focus on the parameter estimation of the bilinear digital system by the DE optimal algorithm. The purpose is to seek for the actual bilinear digital system parameters using the evolutionary operations of the algorithm. Hence, in the beginning all system parameters need to be collected as a parameter vector, and many such parameter vectors then form a population of the algorithm. In the DE algorithm, there are three main evolutionary mechanisms: mutation, crossover, and selection. All parameter vectors in the population are evolved by these three operations to approach the system optimal or near optimal solutions. The rest of this paper can be organized as follows. In Section 2, the difference equation structure 
for the bilinear digital system is clearly described. Section 3 will explain the detailed DE algorithm and apply it to the parameter estimation of bilinear digital system. In Section 4, an illustrative example is provided to show the applicability and robustness of the developed scheme. Finally, a simple conclusion is addressed in Section 5.

\section{Bilinear digital system}

Before introducing the bilinear digital system, let us explain another digital system, the infinite impulse response (IIR) digital filter, which is often used and discussed in the DSP research. The output of IIR digital filter is influenced by both its previous output signals and present and previous input signals. In general, this kind of filter can use fewer coefficients than the finite impulse response (FIR) digital filter to achieve the same filtering purpose. Nevertheless, the stability problem for designing the IIR digital system should always be taken into account. Equation (1) expresses the difference equation structure for IIR digital filter

$$
\begin{aligned}
y[n]=\sum_{k=1}^{N} a_{k} y[n-k]+\sum_{k=0}^{M} b_{k} x[n-k] \\
=a_{1} y[n-1]+a_{2} y[n-2]+\cdots+a_{N} y[n-N] \\
\quad+b_{0} x[n]+b_{1} x[n-1]+\cdots+b_{M} x[n-M],
\end{aligned}
$$

where $x$ and $y$ denote the system input and output signals, respectively, $a_{k}$ and $b_{k}$ represent the system coefficients, $N$ is the number of past outputs and is also referred to as the system order, and $M$ is the number of the past input signals. Bilinear digital system considered in this study is an extended version of the IIR digital filter and can be described by

$$
\begin{aligned}
& y[n]=\sum_{k=1}^{N} a_{k} y[n-k]+\sum_{k=0}^{M} b_{k} x[n-k] \\
& +\sum_{k_{1}=0}^{M} \sum_{k_{2}=1}^{N} c_{k_{1} k_{2}} x\left[n-k_{1}\right] y\left[n-k_{2}\right] \\
& =a_{1} y[n-1]+\cdots+a_{N} y[n-N]+b_{0} x[n]+\cdots+b_{M} x[n-M] \\
& +c_{01} x[n] y[n-1]+\cdots+c_{0 N} x[n] y[n-N] \\
& +c_{11} x[n-1] y[n-1]+\cdots+c_{1 N} x[n-1] y[n-N]+\cdots
\end{aligned}
$$

$$
+c_{M 1} x[n-M] y[n-1]+\cdots+c_{M N} x[n-M] y[n-N]
$$

where $c_{k_{1} k_{2}}$ is also the system coefficient which corresponds to the product of the input and output signals.

For convenience and the use of the DE algorithm, Eq. (2) can further be rewritten as a vector form

$$
y[n]=\Theta U^{T},
$$

where $\Theta$ is a collection of all the system coefficients defined by

$$
\begin{aligned}
& \Theta=\left[\theta_{1}, \theta_{2}, \cdots, \theta_{K}\right] \\
& =\left[a_{1}, \cdots, a_{N}, b_{0}, \cdots, b_{M}, c_{01}, \cdots, c_{M N}\right],
\end{aligned}
$$

with the vector length $K=N+(M+1)+(M+1) N$ $=(M+1)(N+1)+N$, and $U$ is another collection of all output, input, and their product signals given by

$$
\begin{aligned}
U=[y[n-1], \cdots, y[n-N], x[n], \cdots, x[n-M], \\
x[n] y[n-1], \cdots, x[n-M] y[n-N]] .
\end{aligned}
$$

For the parameter estimation problem of bilinear digital system, the DE algorithm is utilized to optimally seek for the actual parameter vector of Eq. (4).

\section{DE-based parameter estimations}

As described in previous section, the DE algorithm is composed of three principal operations including mutation, crossover, and selection. This section will clearly explain these operations $^{(1)}$. At the beginning of the algorithm, a cost function of the system needs to properly be defined for evaluating the performance of each parameter vector. It is here given by

$$
C F=\sum_{n=0}^{L} e^{2}[n]=\sum_{n=0}^{L}[y[n]-\hat{y}[n]]^{2},
$$

where $L$ represents the sampling number and $e$ is the error signal between the actual system output $y$ and estimated model output $\hat{y}$. A parameter vector with less cost function stands for a better one. With the definition of cost function in Eq. (6), the following operations are executed for each parameter vector inside the population. Here the population size is denoted by $P S$.

In the mutation operation, a new mutated vector 
$V=\left[v_{1}, v_{2}, \cdots v_{K}\right]$ is obtained using Eq. (7)

$$
V=\Theta_{\alpha}+F \cdot\left(\Theta_{\beta}-\Theta_{\gamma}\right)
$$

where $\Theta_{\alpha}, \Theta_{\beta}$, and $\Theta_{\gamma}$ are three different parameter vectors randomly chosen from the population and $F \in[0,2]$ is a mutation constant factor that controls the amplification of the differential variation $\Theta_{\beta}-\Theta_{\gamma}$. Eq. (7) reveals that the mutated vector $V$ is a full combination of $\Theta_{\alpha}, \Theta_{\beta}$, and $\Theta_{\gamma}$. The derived mutated vector $\mathrm{V}$ will further cross with a target vector $\Theta=\left[\theta_{1}, \theta_{2}, \cdots, \theta_{K}\right]$. In the crossover operation, it is to interchange some elements between mutated vector $V$ and target vector $\Theta$. In order to achieve that, a new vector $\left[r_{1}, r_{2}, \cdots, r_{K}\right]$ is generated where $r_{i}$ is a uniformly random number generated from the interval $[0,1]$ for $i=1,2, \cdots, K$, and another set of binary sequence $\left[p_{1}, p_{2}, \cdots, p_{K}\right]$ is derived by Eq. (8)

$$
p_{i}=\left\{\begin{array}{ll}
1, & \text { if } r_{i}<C R \\
0, & \text { otherwise }
\end{array}, \text { for } i=1,2, \cdots, K,\right.
$$

where $C R \in[0,1]$ is the crossover rate and it is always set to 0.5. Based on this binary sequence, a trial vector $W=\left[w_{1}, w_{2} \cdots, w_{K}\right]$ can eventually be obtained by

$$
w_{i}=\left\{\begin{array}{ll}
\theta_{i}, & \text { if } p_{i}=1 \\
v_{i}, & \text { if } p_{i}=0
\end{array}, \text { for } i=1,2, \cdots, K\right.
$$

It is concluded from the crossover formula of Eq. (9) that the trial vector $W$ is a full exchange outcome between $V$ and $\Theta$. Next step is to execute the selection operation on both the trial vector $W$ and the original target vector $\Theta$. In brief, the selection is to keep the excellent parameter vector and discard the bad one. As a result, the cost functions of both $W$ and $\Theta$ need to be calculated. If $C F(W)<C F(\Theta)$, i.e., the trial vector is superior to the target vector, then the algorithm keep this derived trial vector and discard the original target vector, otherwise the target vector still survives in the population and omit the trial vector.

To perform the above three DE operations once is called a generation or an iteration of the algorithm. In general, there are two kinds of conditions to stop the algorithm: the assigned number of iterations $G$ is attained or the cost function derived during the optimization is already met. In this paper, the algorithm stops when the former is satisfied. Finally, Fig. 1 displays the complete system block diagram for parameter estimation of bilinear digital system using the DE algorithm.

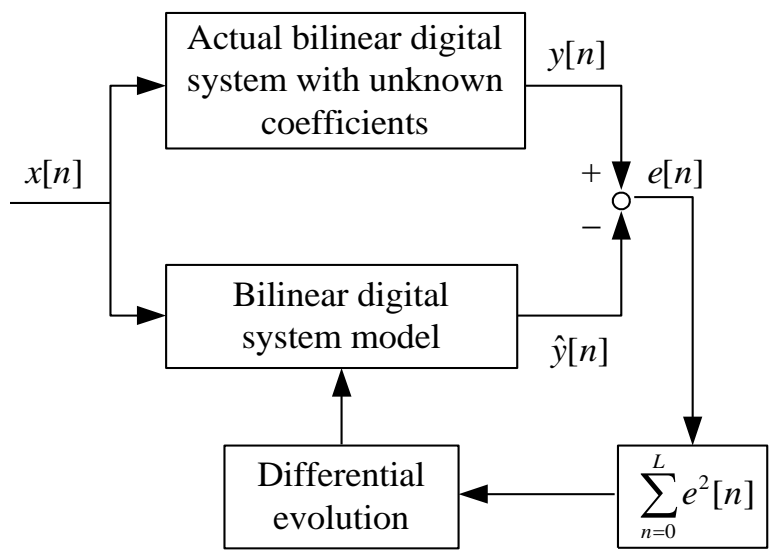

Fig. 1. System block diagram for parameter estimation of bilinear digital system using the DE algorithm.

\section{An illustrative example}

In this section, a simulation example for the parameter estimation of the bilinear digital system is proposed. The bilinear digital system considered can be expressed by

$$
\begin{array}{r}
y[n]=a_{1} y[n-1]+a_{2} y[n-2]+b_{0} x[n]+b_{1} x[n-1] \\
+c_{01} x[n] y[n-1]+c_{11} x[n-1] y[n-1],
\end{array}
$$

where $a_{1}=0.8, a_{2}=-1.2, b_{0}=0.85, b_{1}=-1.72$, $c_{01}=-0.22$, and $c_{11}=0.88$ are given in this study. The related parameters used in the DE algorithm are listed in Tab. 1. The initial value of each parameter vector is randomly generated from the interval $[-1,1]$. Moreover, the input signal $x[n]$ to excite the above bilinear digital system is also chosen from $[-1,1]$ randomly for $0 \leq n \leq 100$. In order to demonstrate the algorithm's robustness, ten independent runs with different sets of initial conditions (Run $1 \sim$ Run 10) are performed for the parameter estimation of the bilinear digital system. Numerical results are listed in Tab. 2 for Run 1 to Run 10. It is clearly seen from Tab. 2 that the final simulation results by any independent run are the same with those of actual 
digital system as shown in Eq. (10). They reveal that the proposed algorithm can accurately estimate the actual system parameters and has a good robustness as well. In addition, the convergence trajectories of all estimated system parameters during the evolution are also displayed with respect to the number of iterations in Figs. 2-5. Figs. 2 and 3 show the convergence trajectories of all estimated parameters for Run 1, and Figs. 4 and 5 are for Run 5, respectively. As can be seen from these figures, all the estimated parameters already approach the actual system parameters about after 500 iterations.

Table. 1. Related parameters used in the DE algorithm.

\begin{tabular}{|c|c|c|c|}
\hline $\begin{array}{c}\text { Number of } \\
\text { iterations } G\end{array}$ & $\begin{array}{c}\text { Sampling } \\
\text { number } L\end{array}$ & $\begin{array}{c}\text { Population } \\
\text { size } P S\end{array}$ & $\begin{array}{c}\text { Mutation } \\
\text { constant } \\
\text { factor } F\end{array}$ \\
\hline 2000 & 100 & 30 & 0.5 \\
\hline
\end{tabular}

Table. 2. Numerical results for Run 1 to Run 10 .

\begin{tabular}{|c|c|c|c|c|c|c|}
\hline & $a_{1}$ & $a_{2}$ & $b_{0}$ & $b_{1}$ & $c_{01}$ & $c_{11}$ \\
\hline Run 1 & 0.8 & -1.2 & 0.85 & -1.72 & -0.22 & 0.88 \\
\hline Run 2 & 0.8 & -1.2 & 0.85 & -1.72 & -0.22 & 0.88 \\
\hline Run 3 & 0.8 & -1.2 & 0.85 & -1.72 & -0.22 & 0.88 \\
\hline Run 4 & 0.8 & -1.2 & 0.85 & -1.72 & -0.22 & 0.88 \\
\hline Run 5 & 0.8 & -1.2 & 0.85 & -1.72 & -0.22 & 0.88 \\
\hline Run 6 & 0.8 & -1.2 & 0.85 & -1.72 & -0.22 & 0.88 \\
\hline Run 7 & 0.8 & -1.2 & 0.85 & -1.72 & -0.22 & 0.88 \\
\hline Run 8 & 0.8 & -1.2 & 0.85 & -1.72 & -0.22 & 0.88 \\
\hline Run 9 & 0.8 & -1.2 & 0.85 & -1.72 & -0.22 & 0.88 \\
\hline Run 10 & 0.8 & -1.2 & 0.85 & -1.72 & -0.22 & 0.88 \\
\hline
\end{tabular}

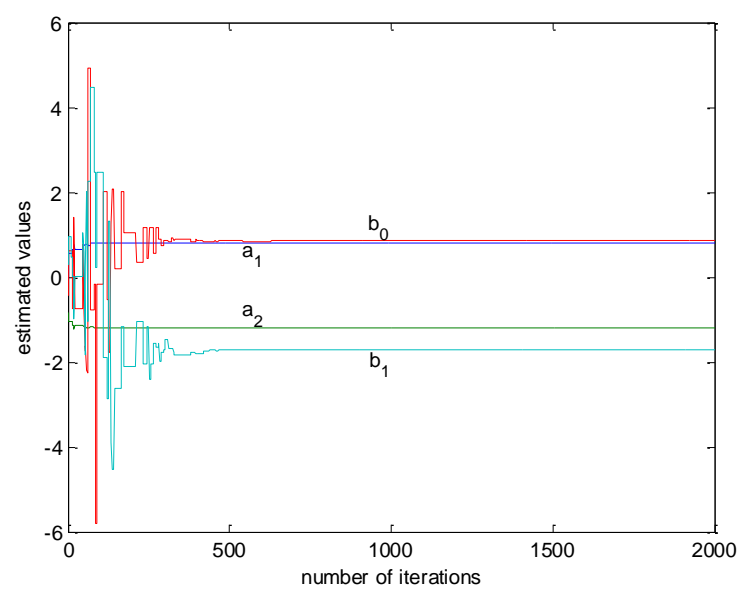

Fig. 2. Convergence trajectories of estimated parameters $a_{1}, a_{2}, b_{0}$, and $b_{1}$ of Run 1 .

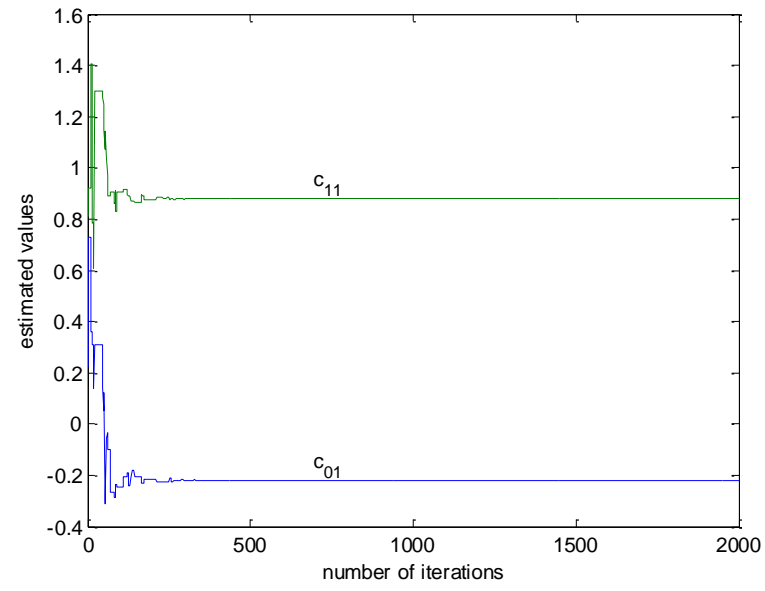

Fig. 3. Convergence trajectories of estimated parameters $c_{01}$ and $c_{11}$ of Run 1.

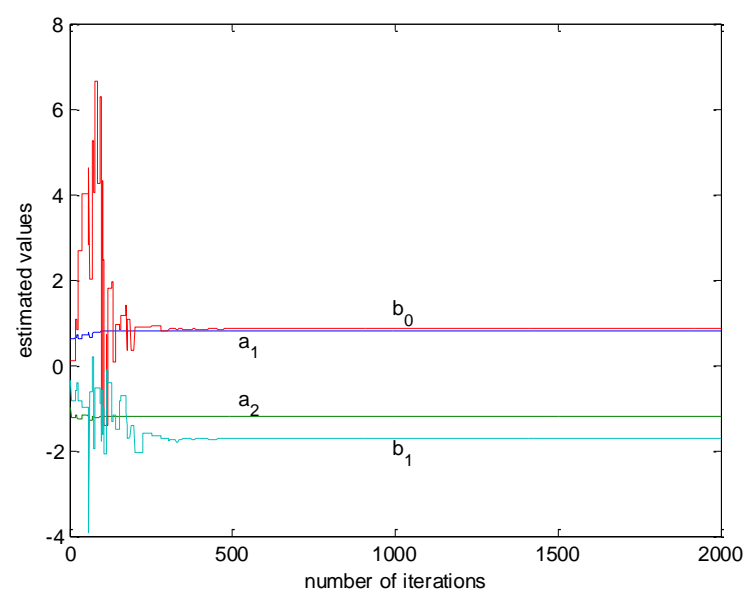

Fig. 4. Convergence trajectories of estimated parameters $a_{1}, a_{2}, b_{0}$, and $b_{1}$ of Run 5 .

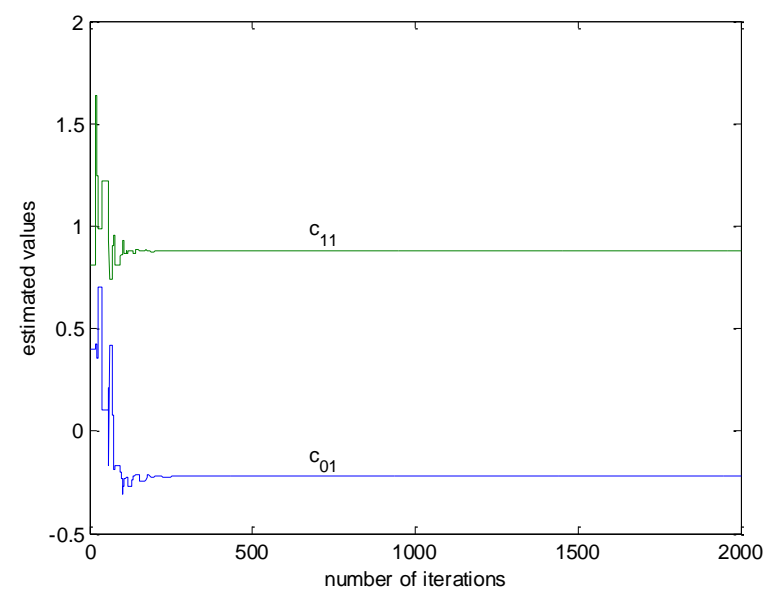

Fig. 5. Convergence trajectories of estimated parameters $c_{01}$ and $c_{11}$ of Run 5. 


\section{Conclusions}

In this paper, we have successfully developed a novel parameter estimation scheme for the bilinear digital system based on using the DE algorithm. All estimated system parameters is collected as a parameter vector of the algorithm. Then, by executing three main evolutionary operations on all parameter vectors inside the population, the actual system parameters can be accurately solved. Furthermore, the robustness of the proposed method is also guaranteed by examining ten independent runs with different sets of initial conditions. Numerical results sufficiently show the applicability of the proposed method on the system parameter estimation of the bilinear digital system. In the future work, other new evolutionary algorithms such as the particle swarm optimization (PSO) and the artificial bee colony (ABC) will be used to solve the same problem and some comparisons are provided.

\section{Acknowledgment}

This work was supported by the Ministry of Science and Technology of Taiwan under the grant MOST 104-2221-E-366-005.

\section{References}

(1) W.D. Chang, Differential evolution-based nonlinear system modeling using a bilinear series model, Applied Soft Computing 12 (2012) 3401-3407.

(2) H. Modares, A. Alfi, M.B.N. Sistani, Parameter estimation of bilinear systems based on an adaptive particle swarm optimization, Engineering Applications of Artificial Intelligence 23 (2010) 1105-1111.

(3) S.K. Singh, A.K. Goswami, N. Sinha, Power system harmonic parameter estimation using bilinear recursive least square (BRLS) algorithm, Electrical Power and Energy Systems 67 (2015) 1-10.

(4) P.L. dos Santos, J.A. Ramos, J.L. Martins de Carvalho, Identification of a benchmark Wiener-Hammerstein: a bilinear and Hammerstein-bilinear model approach, Control Engineering Practice 20 (2012) 1156-1164.

(5) Th.S.S. Singh, A. Chatterjee, A comparative study of adaptation algorithms for nonlinear system identification based on second order Volterra and bilinear polynomial filters, Measurement 44 (2011) 1915-1923.

(6) J. Zhang, H. Zhao, A novel adaptive bilinear filter based on pipelined architecture, Digital Signal Processing 20 (2010) 23-38.

(7) H. Zhao, X. Zeng, Z. He, T. Li, W. Jin, Nonlinear adaptive filter-based simplified bilinear model for multichannel active control of nonlinear noise processes, Applied Acoustic 74 (2013) 1414-1421.

(8) C.M.C. Roque, P.A.L.S. Martins, Differential evolution optimization for the analysis of composite plates with radial basis collocation meshless method, Composite Structures 124 (2015) 317-326.

(9) I. Poikolainen, F. Neri, F. Caraffini, Cluster-based population initialization for differential evolution frameworks, Information Science 297 (2015) 216-235.

(10) S.P. Torres, C.A. Castro, Specialized differential evolution technique to solve the alternating current model based transmission expansion planning problem, Electrical Power and Energy Systems 68 (2015) 243-251.

(11) M. Atif, F.A. Al-Sulaiman, Optimization of heliostat field layout in solar central receiver systems on annual basis using differential evolution algorithm, Energy Conversion and Management 95 (2015) 1-9. 